\title{
Vorhersage und Regelung der Methanproduktion durch maschinelles Lernen
}

\author{
David Wagner ${ }^{1 *}$, Wolfgang Schlüter ${ }^{1}$ \\ ${ }^{1}$ Fakultät Technik, Biomasse-Institut Hochschule Ansbach, Residenzstraße 8, \\ 91522 Ansbach, Germany; ${ }^{*}$ david.wagner@hs-ansbach.de
}

Abstract. Das Anaerobic Digestion Model 1 (ADM1, [1]) ist das umfassendste Modell zur Biogasproduktion. Es enthält 32 dynamische Zustandsgrößen aus reinen Differentialgleichungen (DE) und 26 Zustandsgrößen, sowie 8 algebraischen Variablen, die mittels differential-algebraischen Gleichungen (DAE) beschrieben werden. Es ist daher äußerst flexibel einsetzbar. Das Modell selbst baisert auf Kinetiken erster Ordnung bzw. Monod-Kinetiken. Die Komplexität der Gleichungen führt in Simulationen und Optimierungen häufig zu numerischen Problemen. Um die Biogasproduktion prädiktiv zu steuern, wurden vielfach Modellreduktionen oder -abwandlungen vorgenommen. Darunter leidet die Genauigkeit der Vorhersagen und die Flexibilität. Im folgenden Beitrag wird statt einer Reduktion der Modellstruktur des ADM1 das Modell im vollen Umfang genutzt, um randomisierte Datensätze zu erzeugen. Mithilfe dieser Daten werden maschinelle Lernverfahren trainiert und im Anschluss ein tiefes neuronales Netz (DNN) aufgebaut. Es zeigt eine über 99\%-ige Übereinstimmung zum ADM1 Modell, wenn lediglich Gleichgewichtszustände vorhergesagt werden, und eine $96.7 \%$-ige Übereinstimmung bei Vorhersage zeitlicher Verläufe des Methanstroms $\left(\dot{m}_{\mathrm{CH}_{4}}\right)$. Zudem wird gezeigt, dass das so erhaltene DNN zur Prozesssteuerung verwendet werden kann. Es ist damit in der Lage ein Auswaschen des Reaktors rechtzeitig zu verhindern sowie einen zuvor eingestellten Bedarfsverlauf von $\dot{m}_{\mathrm{CH}_{4}}$ exakt nachzubilden. Damit bietet sich die Möglichkeit zum Einsatz an flexibilisierten Anlagen, die ihre Biogasproduktion am Preis der Strombörse ausrichten.

\section{Einleitung}

Biogas wird durch den anaeroben Abbau von landwirtschaftlichen Substraten, wie Gülle oder Pflanzenresten, hergestellt. Der Prozess gliedert sich in fünf Stufen:
Desintegration (A), Hydrolyse (B), Acidogenese (C), Acetogenese (D) und der Methanogenese (E), den Prozessablauf zeigt Abbildung 1. Mit Ausnahme der Desintegration, katalysieren verschiedene Bakterienkonsortien die jeweiligen Reaktionen.

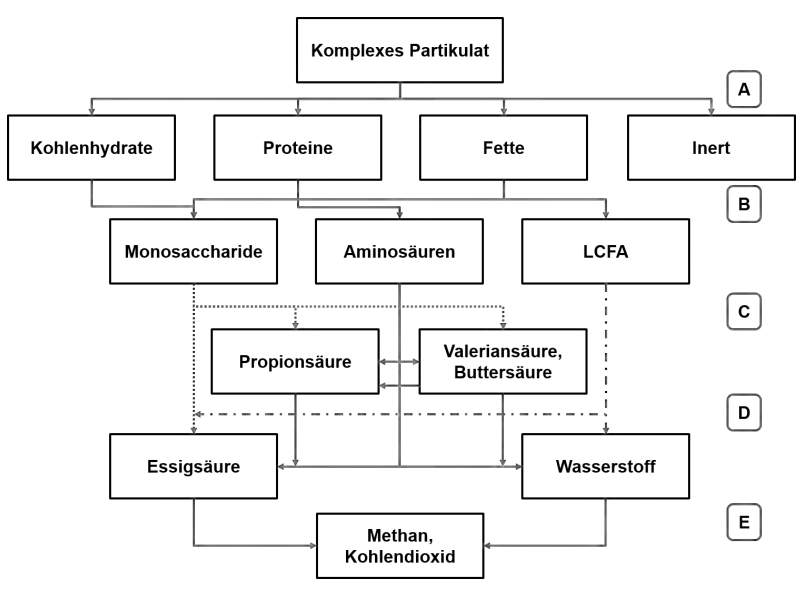

Abbildung 1: Prozess der Biogasproduktion (leicht abgewandelt nach [1]).

Insgesamt werden 105 Parameter für die Beschreibung benötigt. Entsprechende Berechnungen sind langwierig und unterliegen numerischen Instabilitäten. Genaue Parameterkalibrierungen sind mit erheblichem Aufwand verbunden [2, 3]. Für valide Vorhersagen über Gasströme sind diese jedoch erforderlich. Je nach Anlage können die verwendeten Parameter stark vom realen Zustand abweichen. Die eingeschränkte Messbarkeit der Zustandsgrößen limitiert zusätzlich die Identifizierbarkeit der Parameter [4]. Große Konfidenzintervalle und Unsicherheiten im Prozess sind die Folge. Ergebnisse aus Optimierungen unterliegen damit starken Schwankungen. Eine modellprädiktive Regelung (MPC - model predictive control) von Anlagen ist daher mit dem ADM1 nur schwer umsetzbar. Alternativ haben Autoren diverser Publikationen eingeschränkte Modelle oder andere Regler verwendet. So bspw. das Aci- 
dogenese / Methanogenese Modell (AM2) [5] oder ein Modell der Totalalkalinität [6], sowie Regler mit FuzzyLogic [7]. Zwar sind einige Arbeiten der modellbasierten Regelung mithilfe des ADM1 veröffentlicht (Löffler 2012, Cimatoribus 2009, Gaida 2012), allerdings gelten hierbei ebenfalls die vorgenannten Herausforderungen bei der Kalibrierung des ADM1. Weiterhin ist den Autoren keine industriell genutzte Strategie auf Basis des ADM1 bekannt.

Eine Alternative stellen die hier untersuchten BlackBox (statistische) Modelle dar, die den Prozess als solchen außer Acht lassen. Für viele Anwendungen spielt der eigentliche Prozess nur eine untergeordnete Rolle. Für die Prozessregelung sind lediglich die Führungs-, Stell-, Stör- und Regelgrößen relevant. In generischen PID-Reglern wird die Regelgröße in jedem Zeitschritt neu mit der voreingestellten Führungsgröße verglichen und demnach die Stellgröße beeinflusst. Der MPC ist zusätzlich in der Lage, Vorhersagen über den Prozess zu machen. Damit kann die Stellgröße in jedem Zeitschritt bezüglich des gewünschten zukünftigen Regelgrößenverlaufs optimiert werden. Der Vorteil der MPC gegenüber einer PID-Regelung besteht in der prädiktiven Komponente, die kommende Ereignisse, wie bspw. ein Prozessversagen, antizipieren kann. In Abbildung 2 ist diese Form der Regelung schematisch dargestellt. Die Stellgrößen enthalten die manipulierten technischen Parameter im Prozess (Dilution), die Regelgrößen stellen die durch Messwerte belegbaren Prozessausgangsgrößen $\left(\dot{m}_{\mathrm{CH}_{4}}\right)$ dar. Prozessstörungen, durch Messungen oder (unerwünschte) Nebenprozesse, müssen in der Regelung ausgeglichen werden. Der Prozessausgang wird rückgeführt und mit der Führungsgröße (Referenzwert / Idealverlauf) verglichen woraus eine Regelabweichung bestimmt wird. Der Regler selbst kann dabei auf physikalischen oder statistischen Modellen basieren.

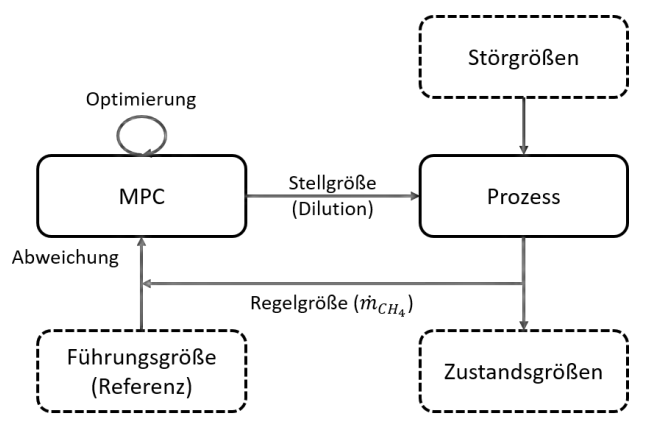

Abbildung 2: Vereinfachtes Schema der MPC-Regelung.

Der MPC-Regler macht Vorhersagen zum Regelgrößenverlauf über einen gleitenden Zeithorizont. Die Op- timierung orientiert sich an einem vorgegebenen Idealverlauf. Um möglich zeitgerecht auf Prozessstörungen zu reagieren, muss die Stellgröße schnell optimiert werden. Die benannten physikalischen Modelle haben den Nachteil, lediglich Subprozesse abzubilden oder durch ihre Komplexität und damit verbundene Trägheit drohendes Prozessversagen nicht zeitgerecht antizipieren zu können. Im Folgenden werden daher diverse maschinelle Lernmethoden und neuronale Netze mit dem Ziel verglichen, das ADM1 akkurat nachzubilden. Die maschinellen Lernmethoden werden anhand verschiedender, durch das ADM1 generierter, Datensätze trainiert. Um zu prüfen welche Prozessgrößen (Stellgrößen) den größten Einfluss auf den Methanmassenstrom (als Regelgröße) haben, wird eine explorative Datenanalyse (EDA) vorgeschaltet. Nachfolgend werden die relevanten Prozessgrößen zufällig variiert und die Simulationsergebnisse als Trainingsdatensätze genutzt. In einem eingeschränkten Datensatz wird die Dilutionsrate (Fütterungsrate) limitiert, so dass es zu keinem Prozessversagen kommen kann. In einem zweiten komplexen Datensatz wird diese Einschränkung gelockert, so dass es zu einem Auswaschen der Biomasse kommt. In einer ersten Analyse werden nur die Gleichgewichtspunkte vorhergesagt. Im Anschluss werden auch Zeitverläufe nachgebildet, die für einen Einsatz im MPC essentiell sind. Dazu wird das maschinelle Lernverfahren ausgewählt, welches die geringsten Abweichungen zeigt (Minimum residualen quadratischen Fehler - $\min (R S S)$ ). In der dargestellten Anwendung dient das Verfahren sowohl zur Regelung des Gleichgewichtszustandes (Endpunktregler), als auch zur kompletten Verlaufsregelung des Methanstroms. Das Vorgehen ist in Abbildung 3 gezeigt.

\section{Material \& Methoden}

\subsection{Datenvorbereitung \& -analyse}

Die Güte maschineller Lernverfahren hängt in erster Linie von der Datenbasis ab. Die Simulation des ADM1Modells erfolgt in Matlab 2019a (auf Basis von [8]). Es werden dafür alle Eingangsparameter im ADM1 variiert. Die Simulationen fließen anschließend in die EDA ein, in der die Korrelation (Corr) zwischen den Eingangsparametern untereinander und dem Gasstrom analysiert wird. Es gilt:

$$
\operatorname{Corr}(x, y)=\frac{\operatorname{Cov}(x, y)}{\sqrt{\operatorname{Var}(x) \operatorname{Var}(y)}}
$$




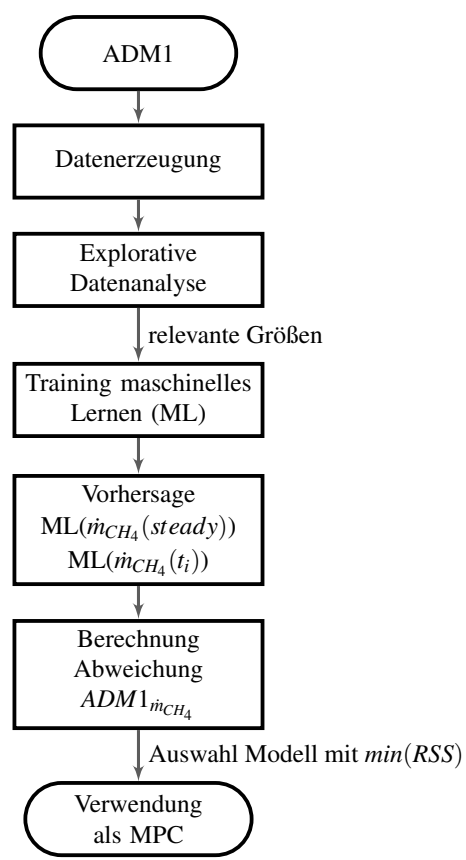

Abbildung 3: Vorgehensschema zur Generierung des MPCReglers.

Die Grenzen der Simulation bilden dabei die theoretischen Parametergrenzen. In Tabelle 1 sind die Wertebereiche der relevanten Eingangsgrößen gezeigt.

\begin{tabular}{l|l|l}
\hline Parameter & $\min$ & $\max$ \\
\hline Dilution (dil) & $1 \mathrm{e}-2 h^{-1}$ & $5 \mathrm{e}-2 / 2 \mathrm{e}-1 h^{-1}$ \\
\hline Kohlenhydratanteil (ch) & $0 \%$ & $100 \%$ \\
\hline Proteinanteil (pr) & $0 \%$ & $100 \%$ \\
\hline Lipidanteil $(l i)$ & $0 \%$ & $100 \%$ \\
\hline
\end{tabular}

Tabelle 1: Randbedingungen für die Simulationsdaten.

Im eingeschränkten Datensatz kommt es unter keinen Umständen zu einem Prozessversagen, da keine Bakterien ausgewaschen werden. Die obere Grenze der Dilution wird auf $5 e-2$ limitiert. Für den komplexen Datensatz ergibt sich unter Umständen ein Auswaschen der bakteriellen Masse und damit ein Einbruch der Gasproduktion. Für beide Datensätze werden insgesamt 12.000 Punkte zufällig innerhalb der Grenzen mittels ADM1 simuliert und der Methanmassenstrom $\left(\dot{m}_{\mathrm{CH}_{4}}\right)$ zeitlich aufgezeichnet. Es wird für die Datenerzeugung das Standardsubstrat aus Batstone et al. verwendet, die Substratanteile werden jedoch (proteins $p r$, carbohydrates - $c h$, lipids - li) ebenso wie die Dilutionsrate (dil) variiert.
Die diversen Wertebereiche der Einflussgrößen machen eine Skalierung vor dem Training notwendig.

\subsection{Werkzeuge \& Bibliotheken}

Alle im Folgenden gezeigten Berechnungen finden unter Windows 10 Enterprise (Version 1903) auf einem 64Bit-System mit Core i7-6600 Kern und 2.6 GHz statt. Die Größe des Arbeitsspeichers beträgt 16 GB.

Für die verwendeten Programme und Werkzeuge siehe Tabelle 2. Matlab dient lediglich der Simulation der Daten mit dem ADM1. Andere Operationen finden in Python innerhalb der Anaconda-Umgebung statt. Bei den Regressionen handelt es sich um lineare Regression (LR), polynomische Regression (PR), KernelRidge Regression (KRR) und Random Forest (RF). Bei den polynomischen Regressionen wird ein Polynom 6. Grades verwendet. Die verwendeten neuronalen Netze werden mit NumPy (Ein-Schichten Netz) bzw. Pytorch (Multi-Schichten Netz) aufgebaut. Für die Validierung der einzelnen Modelle werden jeweils $10 \%$ der Daten als Testdatenset zurückgehalten. Trainingsund Testdaten werden dabei zufällig ausgewählt. Alle Optimierungen werden unter denselben Bedingungen in Python durchgeführt. Die Optimierung des ADM1 wird über eine Schnittstelle (Matlab-Kernel) zwischen Python und Matlab realisiert. Die lineare Optimierung wird mit 20 verschiedenen Startwerten aufgerufen und die erhaltenen Ergebnisse miteinander verglichen.

\begin{tabular}{l|l|l}
\hline Programm & Werkzeug & Funktion \\
\hline Matlab (9.6.0) & ode15s-solver & Simulation \\
\hline Python (3.7.1) & scikit-learn (0.22.2) & Regression \\
\hline- & NumPy (1.17.0) & Arrayoperation \\
\hline- & PyTorch (1.4.0) & $\begin{array}{l}\text { Neuronale } \\
\text { Netze }\end{array}$ \\
\hline- & BFGS / shgo-sobol & $\begin{array}{l}\text { Optimierung } \\
\text { lokal / global }\end{array}$ \\
\hline
\end{tabular}

Tabelle 2: Verwendete Werkzeuge bzw. Bibliotheken in Matlab und Python.

\subsection{Neuronale Netze}

Die verwendeten neuronalen Netze lassen sich in zwei grundlegende Topologien einordnen. Im einfachsten Fall ist lediglich eine Schicht enthalten (Verbindung Input- und Output layer - $N N$ ). Es werden nur lineare Beziehungen zwischen Ein- und Ausgabe ermittelt. Um 
die Vorhersagegenauigkeit zu steigern werden schrittweise Hidden Layer zugefügt $\left(H_{n}^{m}\right.$ in Abbildung 4). Diese erhöhen den Grad der Nichtlinearität. Die Stärke (bspw. $w_{1,1}$ ) der Verbindungen zwischen den Knoten determiniert die Aktivierung des Neurons. Die einzelnen Werte fließen in die Gewichtsmatrix $W$ ein. Eine Aktivierung findet statt, wenn die Werte einen Schwellenwert, den bias $(b)$, überschreiten.

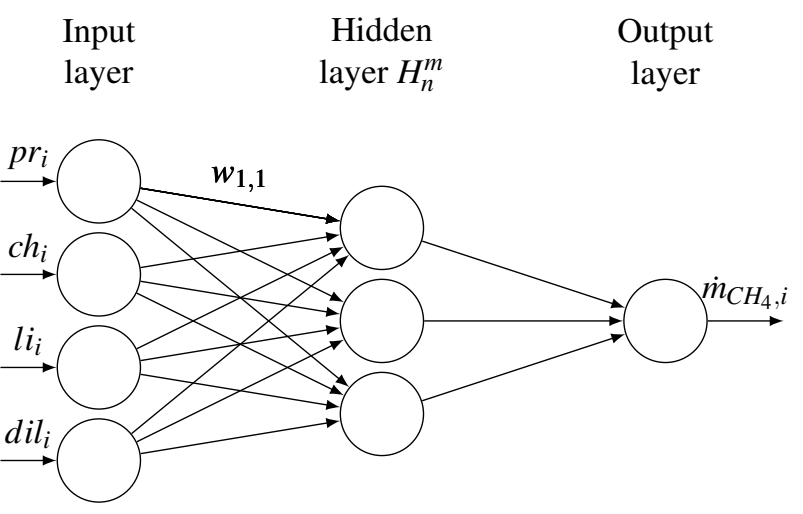

Abbildung 4: Tiefes neuronales Netz zur $\dot{m}_{\mathrm{CH}_{4}}$-Vorhersage.

Die Netze werden durch die Optimierer Adam und SGD (stochastic gradient descent) trainiert. Die Verlustfunktion ist in beiden Fällen der RSS (Gleichung 2). Sie wird ebenso, mit Ausnahme der KRR, auch für die Regressionen bei scikit-learn verwendet.

$$
R S S=\sum_{i=1}^{n}\left(\hat{\dot{m}}_{\mathrm{CH}_{4}, i}-\dot{m}_{\mathrm{CH}_{4}, i}\right)^{2}
$$

Der Index $i$ repräsentiert den jeweiligen Datenpunkt ( $n=12.000)$. Während $\dot{m}_{C H_{4}, i}$ den wahren (durch ADM1 simulierten) Prozessausgang darstellt, handelt es sich bei $\hat{\dot{m}}_{\mathrm{CH}_{4}, i}$ um den modellseitig ermittelten Methanmassenstrom. Für die KRR wird ein Regularisierungsparameter $\alpha$ zur Gleichung 2 hinzugefügt, dieser bestraft die Anzahl der Koeffizienten $c_{p}$ (die Komplexität).

$$
R S S_{k r}=\sum_{i=1}^{n}\left(\left(\hat{\dot{m}}_{C H_{4}, i}-\dot{m}_{C H_{4}, i}\right)^{2}+\alpha\left(c_{p}\right)^{2}\right)
$$

Die in der Optimierung berücksichtigten Parameter sind einerseits die Einträge in der Gewichtsmatrix $W$ und andererseits die bias-Terme $b$. Es ergibt sich damit für den Fall eines Input- und eines Output-layers fol- gende Gleichung:

$$
\hat{\dot{m}}_{C H_{4}, i}=w_{1,1} l i_{i}+w_{1,2} c h_{i}+w_{1,3} p r_{i}+w_{1,4} d i l_{i}+b
$$

Das im Folgenden verwendete tiefe neuronale Netz (DNN) enthält acht Hidden Layer (als $H_{n}^{m}$ in Abbildung 4 dargestellt).

\section{Ergebnisse \& Diskussion}

In dem hier dargestellten Szenario wird ein MPCRegler auf Basis eines Black-Box Modells betrieben. Das ADM1 (im Realfall der Biogasreaktor) entspricht der Regelstrecke, die technischen Parameter (wie z.B. dil) den Stellgrößen und $\dot{m}_{\mathrm{CH}_{4}}$ der Regelgröße. Ein gravierender Nachteil neuronaler Netze ist die mangelnde Extrapolierbarkeit, da ihre Gültigkeit im Allgemeinen auf den Raum der Datenaufzeichnung beschränkt ist. Die mangelnde Extrapolierbarkeit gilt jedoch hierbei ebenfalls für das ADM1. Die Kalibrierung von Parametern muss für jede Anlage neu erfolgen, mit den eingangs beschriebenen Problemen der NichtIdentifizierbarkeit einzelner Parameter und den daraus folgenden Unsicherheiten bei der Optimierung. Trotzdem gelten die hier dargestellten Ergebnisse lediglich als Proof of Principle. Sie zeigen, dass sowohl maschinelle Lernverfahren als auch neuronale Netze die Genauigkeit der Vorhersagen des ADM1 erreichen können. Zudem wird aufgezeigt, dass die Rechenzeiten für die Optimierung deutlich geringer sind. Im Anschluss wird das tiefe neuronale Netz (DNN) genutzt, um mit dessen Hilfe die Dilution entsprechend voreingestellter Idealverläufe des Methanstroms zu optimieren. Wie abschließend gezeigt, lässt sich damit ein Auswaschen der Anlage präventiv verhindern.

\subsection{Datenvorbereitung \& -analyse}

Die Verteilung der skalierten Eingangswerte, die eine gleichmäßige Abdeckung des Raums deutlich macht, ist in Abbildung 5 dargestellt.

Die Skalierung erhöht die Wahrscheinlichkeit der Konvergenz im Trainingsprozess. Es ist allerdings darauf zu achten, dass für eine sinnvolle Aussage eine Reskalierung durchgeführt werden muss. Das Ergebnis der explorativen Datenanalyse ist als Korrelationsmatrix in Abbildung 6 gezeigt.

Es ist deutlich zu erkennen, dass die Dilutionsrate den größten Einfluss auf den Gasfluss $\left(\mathrm{CH}_{4}\right)$ hat. Dies 


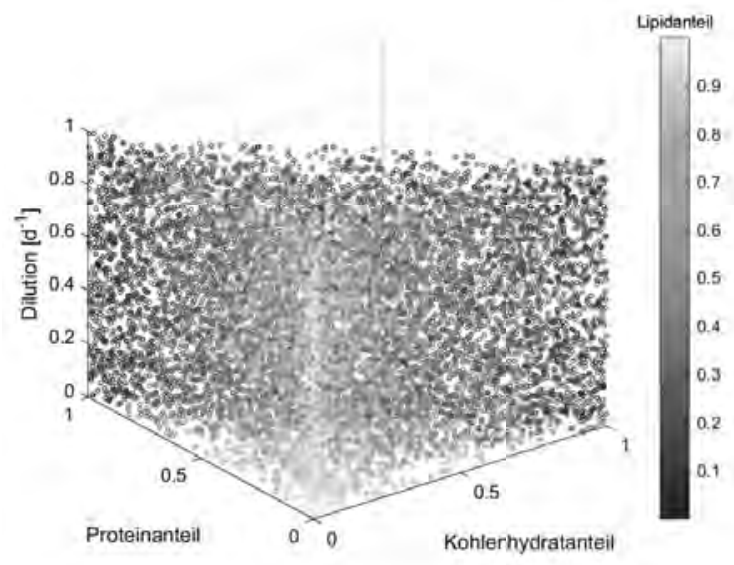

Abbildung 5: Verteilung der Datenpunkte.

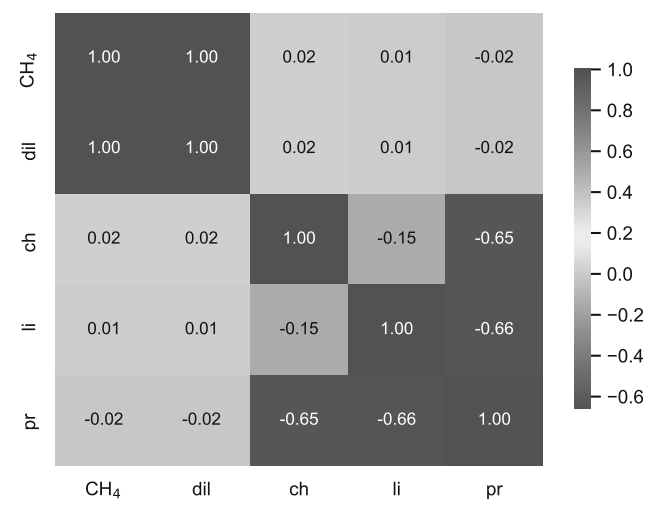

Abbildung 6: Korrelatonsmatrix relevanter Größen.

ist zu erwarten, da die Dilution mit der Fütterungsrate gleichzusetzen ist. Ein hoher Substrateinstrom sorgt für eine gute Versorgung der Bakterien und damit hohen Produktivitätsraten. Andererseits ist die Fütterung weitgehend steril. Daher erfolgt bei Dilutionsraten, jenseits der Bakterienwachstumsraten, ein Auswaschen der Biomasse. Die Korrelationen zwischen Substratanteilen und Gasfluss sind verschwindend gering. Dadurch kann man vermuten, dass bereits lineare Modelle hohe Vorhersagegenauigkeiten erzielen können. Eine Dimensionsreduktion ist jedoch nicht sinnvoll, da die Anteile der Substrate auch direkt mit der einstellbaren Dilution zusammenhängen.

\subsection{Modelldiskriminierung \& -anpassung}

\subsubsection{Simple Regressionsmodelle}

Die verwendeten Regressionsmodelle sind:
- Random Forest (RF) - nichtlinear, multiple Entscheidungsbäume

- Polynomial Regression (PR) - nichtlinear

- Kernel Ridge Regression (KRR) - nichtlinear mit Komplexitätsbestrafung

- Linear Regression (LR) - linear

Im ersten Versuch werden die Modelle dazu genutzt den Gasstrom im Gleichgewicht vorherzusagen. Dabei kommt der vereinfachte Datensatz ohne mögliches Auswaschen zum Einsatz.

\begin{tabular}{l|l|l|l|l}
\hline & RF & PR & KRR & LR \\
\hline Acc $_{\text {Train }}$ in \% & 99.99 & 99.99 & 99.99 & 99.75 \\
\hline $\mathbf{R S S}_{\text {Test }}$ & $3.0 \mathrm{e}-4$ & $1.2 \mathrm{e}-6$ & $1.4 \mathrm{e}-2$ & $2.3 \mathrm{e}-1$ \\
\hline
\end{tabular}

Tabelle 3: Genauigkeit der getesteten Regressionsmodelle auf dem vereinfachten Datensatz.

Tabelle 3 zeigt sowohl die Genauigkeit der Daten auf dem Trainingsset als auch die Abstraktionsfähigkeit anhand der Abweichung zwischen Testdaten und vorhergesagten Gasströmen. Die polynomiale Regression zeigt die geringste Abweichung. RF-Regressionen neigen generell zum Overfitting, weshalb die Abweichung auf den Testdaten geringfügig höher ist, als bei der PR. Die Abweichung bei KRR ist nochmals höher, was vor allem an den zusätzlichen Parametern liegt (vornehmlich von $\alpha$ ). Auch die LR zeigt eine sehr gute Übereinstimmung mit den Testdaten. Aufgrund der vorrangigen Abhängigkeit des Gasstroms von der Dilution ist dieses Ergebnis nicht überraschend. Alle Regressionmethoden sind für den definierten Bereich ohne Auswaschen geeignet. Bei Übertragung auf den größeren Dilutionsbereich im komplexen Datensatz (siehe Tabelle 4) versagen viele jedoch.

\begin{tabular}{l|l|l|l|l}
\hline & RF & PR & KRR & LR \\
\hline Acc $_{\text {Train }}$ in \% & 99.94 & 77.67 & 55.15 & 41.77 \\
\hline $\mathbf{R S S}_{\text {Test }}$ & $2.7 \mathrm{e}-1$ & $4.7 \mathrm{e}+1$ & $9.3 \mathrm{e}+1$ & $1.2 \mathrm{e}+2$ \\
\hline
\end{tabular}

Tabelle 4: Genauigkeit der getesteten Regressionsmodelle auf dem komplexen Datensatz.

Lediglich der RF-Regressor ist in der Lage Genauigkeiten über $99 \%$ sowohl auf den Trainings- als auch Testdaten zu erzeugen. 


\subsubsection{Einfaches neuronales Netz (NN)}

Aufgrund der Ergebnisse bei den Regressionsmethoden, kann davon ausgegangen werden, dass auch ein DNN in der Lage sein wird, die Daten genau vorherzusagen. Im ersten Schritt wird der eingeschränkte Datensatz auf ein neuronales Netz ohne Hidden Layer angewandt. Dieses Konstrukt entspricht einer LR und sollte daher ähnliche Genauigkeiten liefern. In diesem Fall besteht die Gewichtsmatrix lediglich aus einem Vektor mit vier Komponenten, hinzu kommt ein bias-Term. Das Training des Netzes liefert für die Gleichung (4) die folgenden Werte:

$w=\left[\begin{array}{llll}-0.62 & -0.62 & -0.60 & 1.02\end{array}\right], b=[0.68]$

Da bereits die Korrelationsmatrix den Einfluss der Substratzusammensetzungen innerhalb der Nährstoffe annähernd gleich bewertet, ergeben sich (bis auf die Dilution) auch ähnliche Gewichtungsfaktoren.

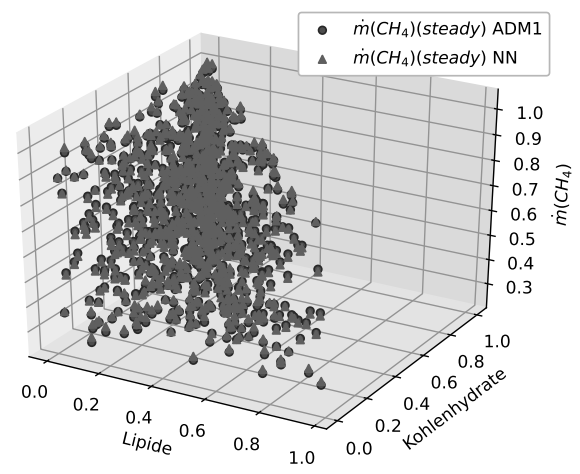

Abbildung 7: Testdaten vereinfachter Datensatz (blau) und Vorhersage durch NN (rot).

Die Genauigkeit mit diesem simplen neuronalen Netz ist dabei sogar der einfachen linearen Regression überlegen. Ein Vergleich zwischen den Testdaten und dem vorhergesagten Gasstrom ist in Abbildung $7 \mathrm{zu}$ sehen. Wird dasselbe Netz auf den komplexen Datensatz angewandt, ergibt sich ein anderes Bild. Einerseits resultiert das Auswaschen in einem nichtlinearen Verhalten des Gasstroms, andererseits sind bei bestimmten Zusammensetzungen aus Substrat und Dilution die Gasströme gleich oder nahe Null. Dieses Verhalten lässt sich mit einem Ein-Schichten Netz nicht wiedergeben (siehe Abbildung 8).

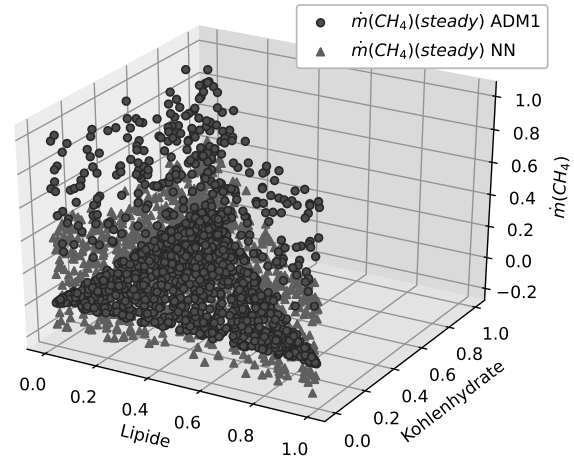

Abbildung 8: Testdaten komplexer Datensatz (blau) und Vorhersage durch NN (rot).

\subsubsection{Tiefes neuronales Netz (DNN)}

Der RF-Regressor kann auch Daten unter Auswaschkonditionen abbilden. Der Grund dafür ist, dass RFRegressoren aus einer Vielzahl Entscheidungsbäumen bestehen. Sie erkennen somit auch komplexere Sachverhalte, wie bspw. welche Kombination aus Dilution und Substratzusammensetzung zu einem Auswaschen führt. Der Nachteil ist die hohe Ressourcennutzung der erhaltenen Modelle und die angesprochene Tendenz zum Overfitten. Aus diesem Grund wurde zusätzlich ein DNN aufgebaut. Das erstellte Netz enthält acht Hidden Layer. Es ergeben sich insgesamt 510.528 Parameter (Gewichtungsmatrix und bias-Terme), die innerhalb des Trainings optimiert werden.

Die Genauigkeit bei der Bestimmung des Methanmassenstroms sowohl für den vereinfachten Datensatz als auch den komplexen Datensatz liegt weit über $99 \%$.

\subsubsection{Verlaufskurvenvorhersage}

Um das DNN im MPC-Kontext nutzen zu können bedarf es zusätzlich noch Informationen über den Zeitverlauf der Methanproduktion. Der Zeitverlauf wird mit 16 Zeit-Massenstrompunkten aufgelöst. Diese gehen in das Training des DNN ein.

Auf den Testdaten beträgt die Genauigkeit 96.70\%. Das DNN ist in der Lage, aus der Dilution und der Substratzusammensetzung, vorherzusagen, ob es zu einem Auswaschen während des Prozesses kommt. Beispielhaft sind in der Abbildung 9 zeitliche Verläufe und deren Übereinstimmung mit den simulierten Daten ohne und mit Auswaschen gezeigt. Diese Werte wurden ohne 
die Optimierung von Hyperparametern, wie bspw. Dropout, Lernrate oder Lernratenabschwächung, ermittelt.

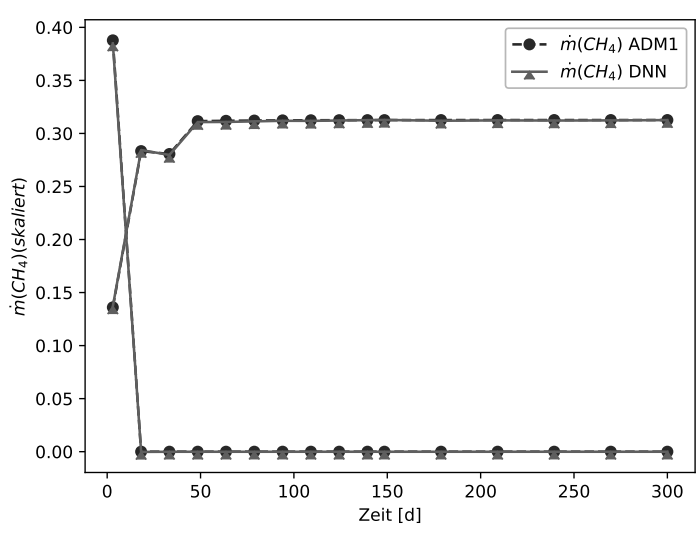

Abbildung 9: Beispielverlauf ohne (obere Kurve) /mit (untere Kurve) Auswaschen. Testdaten blau, Daten von DNN vorhergesagt rot.

\subsubsection{MPC-Regelung}

Nachdem gezeigt werden konnte, dass alle Datensätze adäquat mit dem DNN dargestellt werden können, wird das DNN in einen MPC integriert. Die Funktionsweise ist schematisch in Abbildung 10 gezeigt.

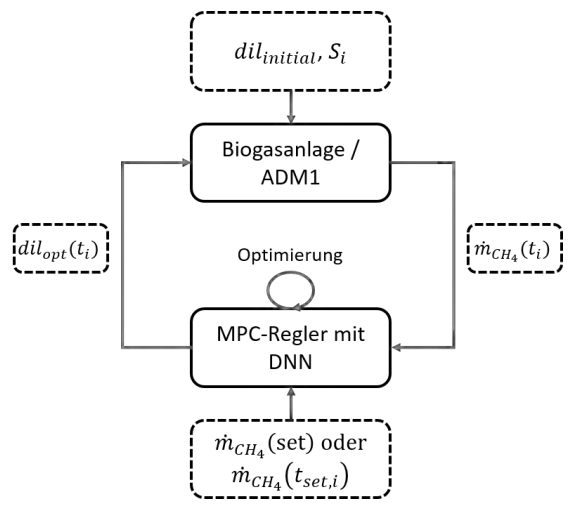

Abbildung 10: Ablauf der MPC-Regelung mittels DNN.

Das ADM1 stellt die Regelstrecke dar und wird mit einer zufällig gewählten Kombination aus Substrat $\left(S_{i}\right)$ und zugehöriger Dilution $\left(\right.$ dil $\left._{\text {initial }}\right)$ initialisiert, um eine beliebige Biogasanlage zu simulieren. In jedem Zeitschritt wird der aktuelle Methanstrom $\left(\dot{m}_{\mathrm{CH}_{4}}\left(t_{i}\right)\right)$, als Regelgröße, an den Regler (das DNN) übermittelt. Daraufhin vergleicht der Regler den derzeitigen Stand mit dem zuvor eingestellten Referenzwert für den Methanmassenstrom. Es ergibt sich eine Regelab- weichung $\varepsilon=\dot{m}_{\mathrm{CH}_{4}}\left(t_{i}\right)-\dot{m}_{\mathrm{CH}_{4}}\left(t_{s e t, i}\right)$. Diese wird mittels Einstellung der durch das DNN optimierten Dilutionsrate $d i l_{\text {opt }}\left(t_{i}\right)$ (Stellgröße) minimiert. Statt zu jedem Zeitschritt, kann die Optimierung auf einen gewünschten Endpunkt $\left(\dot{m}_{\mathrm{CH}_{4}}(\mathrm{set})\right.$ ) erfolgen (hier $\left.1500 \mathrm{~m}^{3}\right)$, wobei es sich dann um einen Endpunktregler handelt und die Dilution lediglich einmal eingestellt bzw. optimiert wird (siehe dazu Abbildung 11).

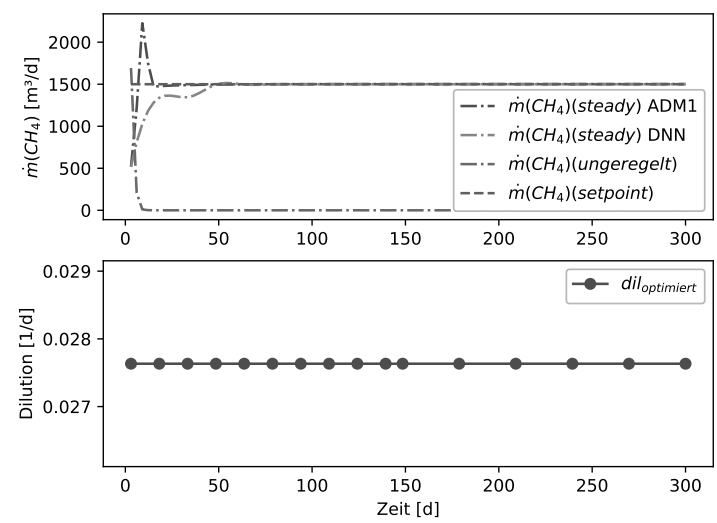

Abbildung 11: Endpunktregelung des Methanstroms. Daten des ADM1 blau, durch das DNN vorhergesagter Verlauf gelb, Referenzwert in rot und ungeregelter Verlauf grün. Unten: Verlauf der Stellgröße.

Um Schwankungen in der Methanproduktion zu verhindern und möglichst auch Überproduktion zu vermeiden, wie in Abbildung 11 sichtbar, kann der MPC die Dilution auch zu jedem Zeitschritt optimieren. Durch die Anpassung der Dilutionsrate kann die Methanproduktion an einen zuvor bestimmten Idealverlauf angeglichen werden (siehe dazu Abbildung 12).

Aus Abbildung 12 lässt sich deutlich entnehmen, dass eine zuvor eingestellte Trajektorie des Methanmassenstroms durch den Regler verfolgt werden kann. Das typische Überschwingen, wie aus der Anwendung von PID-Reglern bekannt, bleibt aus. Außerdem wird ein Prozessversagen ohne die Regelung (siehe grüner Verlauf Abbildung 11) vermieden. Die Rechenzeit für die Ermittlung der optimalen Dilutionsrate (globales Minimum) beträgt bei Verwendung des DNN durchschnittlich lediglich $2 s$, bei der lokalen Optimierung $0.5 s$ pro Optimierung. Zum Vergleich werden dieselben Optimierungen mit dem ADM1 aus Python angestoßen, wobei allein die lokale Optimierung durchschnittlich $633 \mathrm{~s}$ reine Rechenzeit benötigt. 

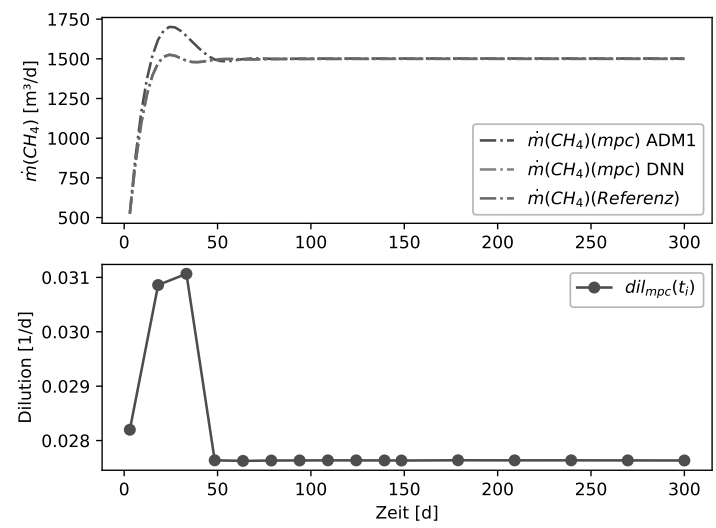

Abbildung 12: Kontinuierliche Regelung des Methanstroms. Daten des ADM1 blau, durch das DNN vorhergesagter Verlauf gelb, Referenzverlauf rot. Unten: Verlauf der Stellgröße.

\section{Schlussfolgerung \& Ausblick}

Diese Veröffentlichung zeigt, maschinelle Lernmethoden können komplexe physikalische Modelle ersetzen. Selbst Verlaufskurven von physikalischen Größen lassen sich mit einfachsten so nachbilden. Die Verfahren können in eine Regelung integriert werden, die ohne Kalibirerungsmaßnahmen auskommt. Für Biogasanlagen stehen die benötigten Trainingsdaten zumeist durch die kontinuierliche Überwachung der Gasmengenzusammensetzung und der zugeführten Substrate zur Verfügung, wohingegen eine Kalibrierung der Modellparameter für das ADM1 zeit- und messintensiv ist. Ein neuronales Netz ist, einmal trainiert, imstande, Optimierungen zeitnah durchzuführen. Dies ermöglicht die Anwendung in einem MPC-Regler, um Methanstromtrajektorien nachzubilden und ein Prozessversagen zu verhindern. Durch einen solchen MPC-Regler lassen sich Fahrpläne, die den ökonomischen Vorgaben der Strombörse folgen, realisieren. Allein aus Zeitgründen sind die dafür erforderlichen Optimierungen mit dem ADM1 nicht realistisch umsetzbar.

\section{Literatur}

[1] D.J. Batstone, J Keller, I Angelidaki, S.V. Kalyuzhnyi, S.G. Pavlostathis, A Rozzi, W.T.M. Sanders, H Siegrist, and V.A. Vavilin. The IWA Anaerobic Digestion Model No 1 (ADM1). Water Science and Technology, 45(10):65-73, 2002.
[2] D. Poggio, M. Walker, W. Nimmo, L. Ma, and M. Pourkashanian. Modelling the anaerobic digestion of solid organic waste - Substrate characterisation method for ADM1 using a combined biochemical and kinetic parameter estimation approach. Waste Management, 53:40-54, 2016.

[3] S. Astals, M. Esteban-Gutiérrez, T. FernándezArévalo, E. Aymerich, J.L. García-Heras, and J. Mata-Alvarez. Anaerobic digestion of seven different sewage sludges : A biodegradability and modelling study. Water Research, 47(16):6033 - 6043, 2013.

[4] Lei Xue, Dewei Li, and Yugeng Xi. Nonlinear model predictive control of anaerobic digestion process based on reduced ADM1. 2015 10th Asian Control Conference: Emerging Control Techniques for a Sustainable World, ASCC 2015, 2015.

[5] Olivier Bernard, Zakaria Hadj-Sadok, Denis Dochain, Antoine Genovesi, and Jean Philippe Steyer. Dynamical model development and parameter identification for an anaerobic wastewater treatment process. Biotechnology and Bioengineering, 75(4):424-438, 2001.

[6] H. O. Méndez-Acosta, B. Palacios-Ruiz, V. Alcaraz-González, V. González-Álvarez, and J. P. García-Sandoval. A robust control scheme to improve the stability of anaerobic digestion processes. Journal of Process Control, 20(4):375-383, 2010.

[7] A. Puñal, L. Palazzotto, J. C. Bouvier, T. Conte, and J. P. Steyer. Automatic control of volatile fatty acids in anaerobic digestion using a fuzzy logic based approach. Water Science and Technology, 48(6):103110, 2003.

[8] C Rosen, D Vrecko, K V Gernaey, M N Pons, and U Jeppsson. Implementing ADM1 for plant-wide benchmark simulations in Matlab / Simulink. Water Science and Technology, 54(4):11-19, 2006. 